\title{
Notwendigerweise unvollkommen: Strukturprobleme des Europäischen Binnenmarktes
}

\author{
von Susanne K. Schmidt
}

Den Binnenmarkt kennzeichnen noch vielfältige Schwierigkeiten, wie nicht zuletzt die Diskussion um die Dienstleistungsrichtlinie zeigt. Der Beitrag untersucht die unterschiedlichen Integrationsprinzipien - Inländergleichbehandlung, Harmonisierung und gegenseitige Anerkennung - und diskutiert damit verbundene Funktionsprobleme. Gerade für Dienstleistungen, so die These, kann der Binnenmarktansatz sein Ziel nur unvollkommen verwirklichen. Angesichts des vielfältigen Regulierungsbedarfs lässt sich in einer sich stetig erweiternden Union nicht verhindern, dass die Grenzen der Pflicht zur gegenseitigen Anerkennung und des Rechts zur Inländergleichbehandlung umstritten bleiben. Die Harmonisierung sämtlicher Regulierungsfragen ist aber keine Alternative. ${ }^{1}$

The single market is still characterised by many problems as current discussions on the directive for services indicate. This article aims at analysing the different integration principles - national treatment, harmonisation, and mutual recognition - and discusses a number of functional deficits. It argues that, particularly for services, the objective of the single market approach may only be realised imperfectly. Given the manifold reasons for regulations, the boundaries between the duty to mutual recognition and the right to national treatment remain necessarily contentious in a continuously enlarging Union. The harmonisation of all controversial issues, however, is no option either.

\section{Einleitung}

Mit dem Plan Mitte der 1980er Jahre, den Binnenmarkt zu verwirklichen, nahm die europäische Integration einen unerwarteten Aufschwung. Schon vor dem offiziellen Datum der Realisierung des Binnenmarktes (Ende 1992) wurden die Pläne zur Währungsunion konkret. Parallel zu ihrer Ausarbeitung liefen die Vorbereitungen der bisher größten Erweiterungsrunde 2004; ganz zu schweigen von der Erweiterung um Finnland, Österreich und Schweden 1995, die im Bemühen um

1 Dieser Aufsatz beruht auf den Ergebnissen meiner Habilitationsschrift, Schmidt, S.: Rechtsunsicherheit statt Regulierungswettbewerb: Die nationalen Folgen des europäischen Binnenmarkts für Dienstleistungen, FernUniversität Hagen, 2004. Ich danke Fritz Scharpf für seine hilfreichen Kommentare zu diesem Projekt und Ines Läufer für Forschungsassistenz. Mein Dank gilt auch den zwei anonymen Gutachtern für ihre sehr konstruktiven Anregungen. 
den Europäischen Wirtschaftsraum ihren Anfang nahm. Gleichzeitig erfolgten verschiedene Vertragsrevisionen - zunächst die Einheitliche Europäische Akte, dann Maastricht, Amsterdam und Nizza und schließlich die Ausarbeitung des Verfassungsvertrages.

Betrachtet man diese beeindruckende Entwicklung, scheint in der Tat der Binnenmarkt vergleichsweise unproblematisch. Demgemäß hat sich auch die politikwissenschaftliche Forschung der letzten Zeit vergleichsweise wenig mit dieser Thematik befasst. Gut ein Jahrzehnt nach der offiziellen Verwirklichung wird aber zunehmend deutlich, dass auch der Binnenmarkt noch vielfältige Probleme birgt, insbesondere was Dienstleistungen betrifft. So ist die Realisierung des Dienstleistungsbinnenmarktes zentraler Bestandteil der europäischen Agenda 2010 der Strategie von Lissabon. Der Vorschlag einer Dienstleistungsrichtlinie, die den Dienstleistungsbinnenmarkt auf einen Schlag verwirklichen soll, ist aber insbesondere in Deutschland sehr umstritten. Gleichzeitig ist die Europäische Kommission selbst mit dem Funktionieren des Binnenmarktes für Waren unzufrieden und überlegt, inwieweit hier zusätzliche regulative Maßnahmen notwendig sind. Schließlich hat nach der Osterweiterung die ohne Übergangsfrist geltende Dienstleistungsfreiheit gerade in Deutschland kürzlich für politischen Aufruhr gesorgt, da sich hiermit jene „Verdrängung“ deutscher Arbeitsplätze durch Osteuropäer anbahnt, die man mit einer Übergangsfrist für die Freizügigkeit eigentlich verhindern wollte. ${ }^{2}$

Angesichts dessen scheint es lohnend, die Funktionsprinzipien des Binnenmarktes näher zu untersuchen. Nach diesem ersten Schritt soll im Folgenden auf die Besonderheiten eines Handels mit Dienstleistungen eingegangen werden. Hiernach werden offene Fragen der Mindestharmonisierung analysiert und die Schwierigkeiten des Binnenmarktes zunächst für Waren und dann für Dienstleistungen geschildert. Verschiedene Beispiele demonstrieren, dass für den Dienstleistungsbinnenmarkt eine Mischung der verschiedenen Integrationsprinzipien typisch ist. Gerade in diesem Bereich, so die These, vermag der Ansatz der gegenseitigen Anerkennung den Binnenmarkt nur um den Preis seiner Unvollkommenheit zu verwirklichen. Angesichts des vielfältigen Regulierungsbedarfes von Dienstleistungen lässt sich gerade in einer sich stetig erweiternden Union nicht verhindern, dass die Grenzen der Pflicht zur gegenseitigen Anerkennung und des Rechts zur Inländergleichbehandlung umstritten bleiben. Die Harmonisierung sämtlicher strittiger Regulierungsfragen bildet aber keine Alternative. Die Flexi-

2 Dies zeigt sich beispielsweise im outsourcing von Schlachthofdienstleistungen an osteuropäische Dienstleistungsanbieter, die zur Arbeitslosigkeit mehrerer Tausend Mitarbeiter führte, vgl. Der Spiegel vom 14. 02. 2005, 32-35. 
bilität der gegenseitigen Anerkennung geht notwendigerweise mit offenen Fragen über ihre genauen Grenzen einher. Deshalb ist die Integration des Binnenmarkts über die gegenseitige Anerkennung unvollkommen, aber zugleich unverzichtbar.

\section{Integrationsprinzipien}

Will man hoch regulierte Märkte ohne eine gleichzeitige politische Vergemeinschaftung integrieren, ist fraglich, wie man mit diesen Regulierungen umgeht. ${ }^{3}$ Ein traditioneller Weg besteht in der Inländergleichbehandlung. Dadurch wird es möglich, in anderen Mitgliedstaaten Dienstleistungen anzubieten, jedoch nur, wenn die dort geltenden Vorschriften eingehalten werden. Wie Abbildung 1 zeigt, bleiben die Märkte aufgrund der Regulierungsunterschiede getrennt. Produkte sind einheitlich national definiert, und es ist zunächst nicht erkennbar, ob es sich um in- oder ausländische Anbieter handelt. Die Harmonisierung führt dagegen zu einer stärkeren Integration von Märkten auf der Basis einer grenzüberschreitenden, einheitlichen Regulierung. Hier geht also auch - punktweise - die politische Integration mit der wirtschaftlichen einher. Bei der gegenseitigen Anerkennung schließlich wird von der Gleichwertigkeit unterschiedlicher Regulierungen ausgegangen. Nationale Märkte werden nicht mehr durch nationale Regulierungen definiert. Somit wird die Trennung zwischen den Märkten aufgehoben, indem unterschiedlich regulierte Produkte gegenseitig anerkannt werden.

Bei der Inländergleichbehandlung sind die Mitgliedstaaten weiterhin frei, die in ihrem Gebiet gehandelten Waren und Dienstleistungen selbst zu regulieren, ihre Souveränität bleibt intakt. ${ }^{4}$ Die politischen Interessen an Regulierung werden aber auf Kosten der Vorteile des Freihandels gewahrt, da ausländischen Unternehmen Anpassungskosten aufgebürdet werden. Inwieweit ist die Inländergleichbehandlung im Rahmen der europäischen Integration relevant? Ursprünglich wurden die Grundfreiheiten des Vertrags als Diskriminierungsverbot interpretiert. Ausländer durften nicht diskriminiert werden, sie mussten sich aber nach den relevanten inländischen Regeln richten. Es galt also die Inländergleich-

3 Vgl. Nicolaïdis, K.: Mutual Recognition Among Nations: The European Community and Trade in Services, PhD Thesis, Harvard University, Cambridge, Mass., 1993, 488-491, 495. Siehe auch die Diskussion in Maduro, M. P.: We the Court. The European Court of Justice and the European Economic Constitution, Oxford, 1998, 101-149, der von Zentralisierung (Harmonisierung), Wettbewerb (gegenseitiger Anerkennung) und Dezentralisierung (Inländergleichbehandlung) spricht.

4 Nicolaïdis, K., a.a. O., 490-493. 
Abbildung 1: Verschiedene Prinzipien der Marktintegration

\begin{tabular}{|c|c|c|c|c|c|c|}
\hline \multicolumn{7}{|c|}{$\begin{array}{l}\text { 1. Inländergleichbehandlung } \\
\text { (Tätigkeitslandprinzip) }\end{array}$} \\
\hline \multicolumn{3}{|c|}{ Land A } & \multicolumn{4}{|c|}{ Land B } \\
\hline$\ddot{8}$ & $x 818$ & $\ddot{8}$ & 0 & $\circ \circ c$ & $\bigcirc$ & 0 \\
\hline$\because$ & 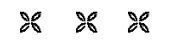 & $\ddot{8}$ & $\bigcirc$ & $\circ 0 c$ & $\bigcirc$ & $\bigcirc$ \\
\hline 88 & $\ddot{8} 8 x$ & $\therefore$ & $\bigcirc$ & $\circ \circ c$ & $\bigcirc$ & O \\
\hline \multicolumn{7}{|c|}{ 2. Harmonisierung } \\
\hline \multicolumn{3}{|c|}{ Land A } & \multicolumn{4}{|c|}{ Land B } \\
\hline & $* * * *$ & & $*$ & $* * *$ & * & \\
\hline$*$ & $* * * *$ & & $*$ & $* * *$ & * & * \\
\hline$*$ & $* * * *$ & * & $*$ & $* * *$ & * & $*$ \\
\hline \multicolumn{7}{|c|}{$\begin{array}{l}\text { 3. Gegenseitige Anerkennung } \\
\text { (Herkunftslandprinzip) }\end{array}$} \\
\hline \multicolumn{3}{|c|}{ Land A } & \multicolumn{4}{|c|}{ Land B } \\
\hline 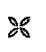 & $0: 80$ & $\ddot{x}$ & $\bigcirc$ & $8 \%$ & $\bigcirc$ & 0 \\
\hline$\therefore$ & $\because 08$ & $\ddot{x}$ & 0 & 008 & $x$ & 0 \\
\hline$\bigcirc$ & $0 \not 8 x$ & 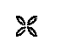 & 28 & $\because 0$ & $\bigcirc$ & 0 \\
\hline
\end{tabular}

Quelle: Schmidt, S., a. a. O., 56.

Anmerkungen: $\mathfrak{g}=$ Produkt gemäß Regulierung Land A. $\bigcirc=$ Produkt gemäß Regulierung Land B. $*$ = Produkt gemäß harmonisierter Regulierung.

behandlung. Bis Anfang der 1980er Jahre hing der Gemeinsame Markt nach dieser Interpretation deshalb vom Gelingen der Harmonisierung ab.

In diesem Fall wird die bisherige nationale durch eine einheitliche supranationale Regulierung ersetzt. Bereits angeglichenes Recht muss auch von der Gemeinschaft fortentwickelt werden. Die Harmonisierung beschränkt sich in der Regel nicht auf grenzüberschreitende Sachverhalte. Stattdessen wird in der EU ein einheitlicher Ansatz verfolgt, so dass Unternehmen bei der Aufnahme grenzüberschreitender Tätigkeiten keine zusätzlichen Regulierungserfordernisse erfüllen müssen. ${ }^{5}$ Harmonisierung entlastet Unternehmen von den Anpassungskosten an unterschiedliche Regulierungen in verschiedenen Mitgliedstaaten, sie belastet aber 
die Regierungen mit dem Einigungsproblem und führt zu einem Souveränitätstransfer auf die supranationale Ebene. ${ }^{6}$ Aufgrund des einheitlichen Ansatzes umfasst der Souveränitätstransfer auch die Regulierung inländischer Sachverhalte.

Die Harmonisierung bezieht sich immer nur auf die Setzung von Regeln, da die Gemeinschaft über keinen eigenen Verwaltungsunterbau verfügt. Die Durchsetzung vergemeinschafteter Regulierung wird deshalb den Mitgliedstaaten überantwortet, in der Regel nach dem Herkunftslandprinzip. Schon bevor sie bei der Regelsetzung eingeführt wurde, kannte die Gemeinschaft die gegenseitige Anerkennung in der Kontrolle harmonisierter Regeln.

Die gegenseitige Anerkennung schließlich ist das Integrationsprinzip, das das Binnenmarktprogramm von 1985 geprägt hat. Statt der mühsamen Totalharmonisierung sollten die Mitgliedstaaten ihre unterschiedlichen Regulierungen in der Regel gegenseitig anerkennen. Nur wo dies unmöglich war und die Mitgliedstaaten ein besonderes Schutzbedürfnis geltend machen konnten, wurde eine Mindestharmonisierung notwendig, die eine Behinderung des Binnenmarktes durch unterschiedliche nationalstaatliche Politiken - nach dem Tätigkeitslandprinzip - verhindert.

Grundlegend für die gegenseitige Anerkennung als Integrationsprinzip war eine veränderte Interpretation der Grundfreiheiten als Beschränkungsverbot durch den Europäischen Gerichtshof (EuGH) beginnend mit Dassonville ${ }^{7}$ und später Cassis de Dijon ${ }^{8}$. Bei einem Diskriminierungsverbot geht es darum, EUAusländer Inländern gleichzustellen und sie nicht zu diskriminieren. Ein Beschränkungsverbot zielt darüber hinaus. Jetzt können die rechtlichen Regelungen des Tätigkeitslandes inhaltlich überprüft werden: Sind sie angemessen und können als verhältnismäßige Beschränkung der Grundfreiheiten von EU-Ausländern gelten? Der Mitgliedstaat ist also nicht mehr frei, In- und EU-Ausländer nach seinem Ermessen zu regulieren. Von hier bis zur gegenseitigen Anerkennung ist es nicht mehr weit: Durch die inhaltliche Überprüfung der Vorschriften des Tätigkeitslandes wird gleichzeitig relevant, welchen Regeln EU-Ausländer als Produzenten von Waren und Dienstleistungen in ihren Heimatländern bereits unterliegen. Doppelkontrollen sind unverhältnismäßig; um sie zu vermeiden, müssen alle Auflagen des Herkunftslandes gegenseitig anerkannt werden. ${ }^{9}$

6 Nicolaïdis, K., a. a. O., 490-493.

7 EuGH, Urt. vom 11. 07.1974, Rs. C-8/74 (Procureur du Roi/Benoît und Gustave Dassonville), Slg. $1974,837$.

8 EuGH, Urt. vom 20.02.1979, Rs. C-120/78 (Rewe-Zentral AG/Bundesmonopolverwaltung für Branntwein), Slg. 1979, 649.

9 Behrens, P.: Die Konvergenz der wirtschaftlichen Freiheiten im europäischen Gemeinschaftsrecht, in: Europarecht, 27/2 (1992), 145-162; Schneider, H.: Zum Funktionswandel der Grundfreiheiten des EGV und zu seinen Auswirkungen auf das nationale Recht, in: Neue Justiz, 50/10 (1996), 512-515. 
Bei der gegenseitigen Anerkennung können Unternehmen ihren Standort und damit auch das Regulierungsregime, unter dem sie produzieren, wählen. Die gegenseitige Anerkennung gibt das Primat dem Handel. Als Regel gilt im Binnenmarkt das Herkunftslandprinzip, dort liegt die Regulierungszuständigkeit. Damit haben Regulierungsentscheidungen von Staaten Externalitäten für andere Staaten. Regierungen haben einen Anreiz, heimischen Unternehmen möglichst vorteilhafte Bedingungen zu bieten, was zu einem Regulierungswettbewerb führen kann, auch in Form einer gegenseitigen Unterbietung (race to the bottom). Höhere Auflagen gehen auf Kosten der Wettbewerbsfähigkeit der eigenen Unternehmen nicht nur auf dem Heimatmarkt, sondern auch im Ausland. ${ }^{10}$ In ihrem Heimatmarkt können Unternehmen auf ausländische Konkurrenten mit regulierungsbedingten Wettbewerbsvorteilen stoßen (Inländerdiskriminierung).

Verglichen mit den anderen Integrationsprinzipien versucht die gegenseitige Anerkennung quasi die Quadratur des Kreises - Unternehmen werden keine Anpassungskosten aufgebürdet und Regierungen keine Verhandlungs- und Entscheidungskosten. Da aber Staaten die Regulierung ihrer Märkte nicht mehr alleine kontrollieren, ergibt sich ein horizontaler Souveränitätstransfer von Land A zu Land B. Die Entscheidung über Regulierungen fällt nicht mehr notwendigerweise in der Jurisdiktion, in der sich die Folgen dieser Regulierung bemerkbar machen. ${ }^{11}$ Der Staat ist nicht mehr allein für die Vorschriften auf seinem Territorium zuständig und verantwortlich, da durch die Herkunftslandkontrolle manche seiner Verbraucher Transaktionen mit Unternehmen tätigen, die von anderen Mitgliedstaaten aus kontrolliert werden. Diese Verbraucher sind somit von der Politik dieser Staaten betroffen, ohne politisch auf sie Einfluss nehmen zu können. ${ }^{12}$ Der zulassende Staat wird „zur europäischen Instanz ${ }^{\text {“13. }}$.

„Durch die Anerkennung verleiht nämlich das jeweilige Gastland den Maßnahmen des jeweiligen Sitzlandes (Herkunftslandes) extraterritoriale Wirkung; die Zulassung oder die Aufsichtsmaßnahme des Sitzlandes gilt nicht mehr nur in dessen Staatsgebiet, sondern, wenn alle Mitgliedstaaten die Anerkennung vornehmen, im gesamten Gebiet der Gemeinschaft. [...] Der zweite Mitgliedstaat erkennt damit nicht nur Qualifikationen, sondern überhaupt die bereits erfolgte Zulassung an; ja noch weiter, er stimmt

Majone, G.: Market Integration and Regulation: Europe after 1992, in: Metroeconomica, 43/1-2 (1992), 131-156; Gatsios, K./Seabright, P.: Regulation in the European Community, in: Oxford Review of Economic Policy, 5/2 (1989), 37-60.

11 Vgl. Nicolaïdis, K., a. a. O., 488-491, 495; Zürn, M.: Sovereignty and Law in a Denationalised World, in: Appelbaum, R. P/Felstiner, W. L. F./Gessner, V. (Hg.): Rules of Networks. The Legal Culture of Global Business Transactions, Oxford, 2001, 39-71, hier 48.

12 Nicolaïdis, K., a. a. O., 16.

13 Troberg, P.: Dienstleistungen, in: Groeben, H.v.d./Thiesing, J./Ehlermann, C.-D. (Hg.), a. a. O., 14411523, hier 1520, Rz. 4. 
nicht nur dem einzelnen Zulassungsakt eines ersten Mitgliedstaates zu, sondern akzeptiert von vornherein alle künftigen Zulassungen, die andere Mitgliedstaaten unter koordinierten Voraussetzungen in Zukunft aussprechen werden. “14

Dieser Souveränitätstransfer macht die gegenseitige Anerkennung zu einem sehr voraussetzungsvollen Integrationsprinzip. Hieraus erklären sich viele Probleme, mit denen der Binnenmarkt zu kämpfen hat. Wie kommt es zur Geltung der gegenseitigen Anerkennung? Aus der breiten Interpretation der Grundfreiheiten folgt der rechtliche Zwang für die Mitgliedstaaten, von anderen Mitgliedstaaten regulierte Waren und Dienstleistungen gegenseitig anzuerkennen. Alternativ kann auch in Richtlinien näher festgelegt werden, in welchem Rahmen die gegenseitige Anerkennung gilt. Nur zum Teil einigen sich die Mitgliedstaaten also explizit. Meist folgt die gegenseitige Anerkennung aus der Rechtsprechung des EuGH.

Auch wenn die Geltung von Richterrecht nicht von der Zustimmung der Mitgliedstaaten abhängt, ist es sinnvoll, nach den Voraussetzungen dieses Integrationsprinzips zu fragen. Zum einen, weil es sich der EuGH ebenso wenig wie die Europäische Union als Ganzes leisten kann, dauerhaft von den Mitgliedstaaten Zugeständnisse zu fordern, die sie nicht zu geben bereit sind. Zum anderen, weil jeder Freiheit auch Schranken gesetzt sind. So steht der Warenverkehrsfreiheit des Art. 28 EGV die Schranke des Art. 30 EGV gegenüber, der für die Mitgliedstaaten legitime Bereiche politischer Gestaltung beschreibt, in denen sie die Marktfreiheiten nicht akzeptieren müssen. Ähnlich werden die anderen Grundfreiheiten im Vertrag eingegrenzt. Zusätzlich hat der EuGH den Mitgliedstaaten parallel zur Ausweitung seiner Interpretation der Grundfreiheiten als Beschränkungsverbot weitere Ausnahmen zugebilligt, die uneinheitlich bspw. als zwingende Erfordernisse im öffentlichen oder im Allgemeininteresse bezeichnet werden. ${ }^{15}$ Hier gilt das Tätigkeitslandprinzip. Um die damit einhergehende Marktzersplitterung zu vermeiden, soll die europäische Mindestharmonisierung erfolgen. Nur wenn die Mitgliedstaaten die gegenseitige Anerkennung akzeptieren, werden sie darauf verzichten, gegenüber Unternehmen aus anderen Mitgliedstaaten auf nationalen Regulierungen unter diesen Ausnahmen zu beharren und so den Binnenmarkt mit langwierigen Gerichtsverfahren zu belasten.

Nach Majone lassen sich vier Voraussetzungen für die gegenseitige Anerkennung differenzieren: Erstens müssen die Mitgliedstaaten ähnliche öffentliche Zielsetzungen mit ihren Regeln verfolgen (wenn auch mit unterschiedlichen Mitteln); zweitens bedarf es einer komplementären Harmonisierung der notwendigen Gesundheits- und Sicherheitsbestimmungen; drittens muss auch der Wettbewerb

14 Troberg, P.: Niederlassung, a. a. O., 1405, Rz. 16.

15 O'Leary, S./Fernandez-Martin, J.: Judicially-Created Exceptions to the Free Provision of Services, in: Andenas, M./Roth, W.-H. (Hg.): Services and Free Movements in EU Law, Oxford, 2002, 163-195, hier $169 \mathrm{f}$. 
der Regulierer geregelt werden, zumal negative Externalitäten nicht über die gegenseitige Anerkennung vermieden werden können; und viertens kann ein System gegenseitiger Anerkennung nur bei ausreichendem gegenseitigen Vertrauen funktionieren. Hier ist also eine solidarische Handlungsorientierung bzw. eine starke Form von Vertrauen notwendig. ${ }^{16}$ Der horizontale Souveränitätstransfer erscheint nur akzeptabel, wenn die Mitgliedstaaten sicher gehen können, dass er von keiner Seite zum eigenen Vorteil ausgenutzt wird. Auch die Harmonisierung soll dieses gewährleisten. Deshalb sind Harmonisierung und die gegenseitige Anerkennung komplementäre Integrationsmechanismen. ${ }^{17}$

Den Hintergrund der notwendigen breiten Vertrauensbasis bildet der horizontale Souveränitätstransfer, den die gegenseitige Anerkennung bedingt. Er impliziert, dass die politische Repräsentation nicht mehr gegeben ist - für die Regulierung ist nicht mehr das Herkunftsland des Verbrauchers, sondern vor allem das des Produzenten zuständig. Damit geben Staaten ihr Monopol auf den Schutz des öffentlichen Interesses in ihrem Territorium auf. ${ }^{18}$ Gleichzeitig bleiben sie aber

Tabelle 1: Charakteristika der Integrationsprinzipien

\begin{tabular}{|l|l|l|l|}
\hline & $\begin{array}{l}\text { Inländergleichbehandlung } \\
\text { (Nicht-Diskriminierung) }\end{array}$ & Harmonisierung & Gegenseitige Anerkennung \\
\hline $\begin{array}{l}\text { Regulierungs- } \\
\text { prinzip }\end{array}$ & $\begin{array}{l}\text { Regulierung des Tätig- } \\
\text { keitslandes }\end{array}$ & $\begin{array}{l}\text { supranational verein- } \\
\text { heitlichte Regulie- } \\
\text { rung (nationale } \\
\text { Durchsetzung) }\end{array}$ & $\begin{array}{l}\text { Regulierung des Herkunfts- } \\
\text { landes }\end{array}$ \\
\hline Kosten & $\begin{array}{l}\text { Anpassungskosten der } \\
\text { Unternehmen }\end{array}$ & $\begin{array}{l}\text { Verhandlungskosten } \\
\text { der Staaten }\end{array}$ & $\begin{array}{l}\text { Kontrollkosten der Staaten, } \\
\text { Auswahlkosten der Ver- } \\
\text { braucher }\end{array}$ \\
\hline $\begin{array}{l}\text { Politische } \\
\text { Folgen }\end{array}$ & $\begin{array}{l}\text { Kein Souveränitäts- } \\
\text { transfer }\end{array}$ & $\begin{array}{l}\text { Vertikaler Souverä- } \\
\text { nitätstransfer }\end{array}$ & $\begin{array}{l}\text { Horizontaler Souveränitäts- } \\
\text { transfer, Regulierungs- } \\
\text { wettbewerb }\end{array}$ \\
\hline $\begin{array}{l}\text { Schwer- } \\
\text { punkt }\end{array}$ & Primat der Politik & $\begin{array}{l}\text { Evtl. Ausgleich } \\
\text { Handel-Politik }\end{array}$ & Primat des Freihandels \\
\hline $\begin{array}{l}\text { Rechtliche } \\
\text { Entsprechung }\end{array}$ & $\begin{array}{l}\text { Ausnahme vom Vertrags- } \\
\text { recht }\end{array}$ & Sekundärrecht & Marktfreiheiten \\
\hline
\end{tabular}

Quelle: Schmidt, S., a. a. O., 75. 1997, $137 \mathrm{f}$.

17 Majone, G.: Mutual Recognition in Federal Type Systems, in: Mullins A./Saunders C. (Hg.): Economic Union in Federal Systems, Sydney, 1994, 69-84, hier 75, 83.

18 Nicolaïdis, K., a. a. O., 488-491, 495. 
bei Fällen von Regulierungsversagen politisch verantwortlich (vgl. gesamthaft zur Problematik Tabelle 1).

\section{Besonderheiten des Handels mit Dienstleistungen}

Entwickelt wurde das Prinzip der gegenseitigen Anerkennung für die Warenverkehrsfreiheit. Später wurde es auf die Dienstleistungsfreiheit vom EuGH übertragen, und Dienstleistungen bildeten auch einen Schwerpunkt im Binnenmarktprogramm der Europäischen Kommission. Da sie aber nicht wie Waren handelbar sind, ist es wichtig, sich ihrer Besonderheiten zu vergegenwärtigen.

Das geschieht zunächst am besten anhand einer Definition. Dienstleistungen, so Vahlens Großes Wirtschaftslexikon, zeichnen sich durch folgende Wesensmerkmale aus: „Immaterialität (keine Lagerfähigkeit (Vergänglichkeit), keine Transportierbarkeit (Standortgebundenheit), keine Wiederverkäuflichkeit, erforderliches Trägermedium (Mensch oder Objekt)), Synchronität von Produktion und Verbrauch (Kunde muss am Produktionsprozess direkt oder indirekt beteiligt sein), Aufrechterhaltung eines bestimmten Leistungspotentials“. Zwar haben Dienstleistungen oft eine sichtbare Form (die Versicherungspolice, das Werbeplakat), doch liegt das für Dienstleistungen wesentliche Element hinter dieser materiellen Ausprägung (die Risikoberechnung, eine werbewirksame Idee). ${ }^{19}$

Die Besonderheiten wirken sich auch auf die Regulierung von Dienstleistungen aus. Obwohl bei Dienstleistungen im Gegensatz zu Gütern der Entstehungsprozess oft nicht klar vom Endprodukt abgrenzbar ist, ist es sinnvoll, diese Unterscheidung fiktiv zu treffen und zwischen dem Dienstleistungs„Produkt" und dem Moment seiner Erbringung zu unterscheiden. ${ }^{20}$ Anders als Güter sind Dienstleistungen in ihrer Produkteigenschaft meist wenig normiert. Für ihre Regulierung kann man zunächst bestimmte Zulassungserfordernisse spezifizieren. ${ }^{11}$ So wird im Binnenmarkt für die Berufsqualifikation durch die gegenseitige Anerkennung von Berufsabschlüssen - unter bestimmten Vorbedingungen - die Herkunftslandkontrolle eingeräumt. Die Zulassung zu einem bestimmten Beruf in einem Mitgliedstaat impliziert die Erlaubnis, diesen auch in

19 Stichwort „Dienstleistungen“ in: Dichtl, E./Issing, O. (Hg.): Vahlens Großes Wirtschaftslexikon, 2. Aufl., München, 1993.

20 Roth, W.-H.: Die Harmonisierung des Dienstleistungsrechts in der EWG, in: Europarecht, 21/4 (1986), 340-369, hier 348 .

21 Roth, W.-H.: The European Court of Justice's Case Law on Freedom to Provide Services: Is Keck Relevant?, in: Andenas, M./Roth, W.-H. (Hg.), a. a. O., 1-24, hier 16. 
anderen Mitgliedstaaten auszuüben. Teils werden auch bestimmte organisatorische Anforderungen an Dienstleistungserbringer gestellt (bspw. die Gesellschaftsform). Davon zu unterscheiden ist die Regulierung der Berufsausübung bzw. bei Banken und Versicherungen die laufende Aufsicht. Hinzu kommen Regulierungen des Dienstleistungsprodukts. Schließlich kann auch der Vertrieb von Dienstleistungen besonders reguliert sein. Im Unterschied zu Gütern geht es bei Dienstleistungen also oft um Prozess-Standards.

„Ob die Likörfabrik im Falle ,Cassis de Dijon' eine gültige Gewerbegenehmigung besaß, war für die Entscheidung [...] unerheblich [...]. Anders bei der Versicherungspolice: Ihre ,Qualität'steht und fällt mit der Solvenz des Versicherers. Ähnliches gilt bei der ,Qualität' der Pauschalreise (Entwertung, wenn der Veranstalter in Konkurs geht) und bei vielen anderen Dienstleistungen (z. B.: Pläne können nicht genehmigt werden, wenn der Architekt nicht zugelassen ist, auch wenn sie technisch nicht zu beanstanden sind). “ 22

Während bei Gütern der Entstehungsprozess vom Endprodukt klar abgegrenzt werden kann und sie deshalb „geschlossen“ Grenzen überschreiten, kennt der Handel mit Dienstleistungen verschiedene Ausprägungen. Hierbei kommt es darauf an, inwieweit ihr Konsum mit der Erbringung zusammenfällt, oder ob die Erbringung etwa mit Hilfe von Telekommunikationsverbindungen räumlich getrennt werden kann. Im erstbenannten Fall ist mit dem Dienstleistungshandel die Grenzüberschreitung von Produktionsmitteln (Kapital, Arbeit) oder der Konsumenten verknüpft.

Folgende Formen des grenzüberschreitenden Dienstleistungsverkehrs lassen sich differenzieren:

- Bei der aktiven Dienstleistungsfreiheit begibt sich der Dienstleistungserbringer vorübergehend in das andere Land.

- Bei der passiven Dienstleistungsfreiheit begibt sich der Dienstleistungsempfänger in das andere Land.

- Eine weitere Möglichkeit (die uns allerdings nicht interessiert) ist, dass sich sowohl Dienstleistungserbringer als auch -empfänger in einen anderen Mitgliedstaat begeben (z. B. Fremdenführer und Touristen).

- Schließlich gibt es Korrespondenzdienstleistungen, die grenzüberschreitend erbracht werden können, ohne dass Erbringer oder Empfänger das Land wechseln. Diese Möglichkeit besteht etwa für Versicherungs- und Bankdienstleistungen, für Telekommunikation und den Rundfunk. ${ }^{23}$ 
Schließlich muss auf eine weitere Besonderheit hingewiesen werden. Die Dienstleistungsfreiheit des Vertrags fasst in (ex) Art. 60 Abs. 1 die Dienstleistungsfreiheit subsidiär zu den anderen Freiheiten. Die Dienstleistungsfreiheit wird demnach relevant, so die Vorschriften über den freien Waren- und Kapitalverkehr oder über die Freizügigkeit nicht greifen. ${ }^{24}$ Das Dienstleistungskapitel hat den Charakter eines „Auffangtatbestandes“ und betrifft „Tätigkeiten von großer Vielfalt“ ${ }^{25}$. Es erfasst „sämtliche Wirtschaftsvorgänge [...], die sich weder als Warenverkehr noch als Personenverkehr noch als Kapitalverkehr einstufen lassen “.26 Der EWGVertrag verfolgt also einen „spezifischen“ Dienstleistungsbegriff, der nicht auf den „tertiären Sektor“ insgesamt zielt, sondern auf den „Vorgang, bei welchem eine Leistung zwischen Wirtschaftssubjekten erbracht wird, die einander als Gebietsfremde gegenüberstehen".27 Weiterhin sind die Merkmale Grenzüberschreitung und Entgeltlichkeit konstitutiv. ${ }^{28}$ Durch das Merkmal der Entgeltlichkeit fallen manche Leistungen wie etwa staatliche Bildungsleistungen nicht unter die Dienstleistungsfreiheit. Es muss jedoch keine Gewinnerzielungsabsicht vorliegen, es geht nur um „eine Beteiligung am Wirtschaftsleben in einem weiten Sinne“.29 Unanwendbar ist die Dienstleistungsfreiheit auf rein innerstaatliche Sachverhalte. ${ }^{30}$

Im Unterschied zu den anderen Freiheiten zielt die Dienstleistungsfreiheit auf Personen und Produkte, was ihre Anwendung erschwert. ${ }^{31}$ Besonders wichtig ist die Abgrenzung von der Niederlassungsfreiheit. Der Vertrag zieht die Grenze zur Niederlassungsfreiheit, indem der „vorübergehende Charakter“ von Dienstleistungen hervorgehoben wird. Der EuGH beurteilt dies „unter Berücksichtigung ihrer Dauer, ihrer Häufigkeit, ihrer regelmäßigen Wiederkehr und ihrer Kontinuität." 32 Sind Tätigkeiten auf Dauer angelegt, wird deshalb die Niederlassungs-

24 Hatzopoulos, V:: Recent Developments of the Case Law of the ECJ in the Field of Services, in: Common Market Law Review, 37/1 (2000), 43-82, hier 48.

25 Roth, W.-H.: Die Harmonisierung des Dienstleistungsrechts, a. a. O., 342.

26 Seidel, M.: Die Dienstleistungsfreiheit in der neuen Rechtsentwicklung, in: Schwarze, J. (Hg.): Der Gemeinsame Markt - Bestand und Zukunft in wirtschaftsrechtlicher Perspektive, Baden-Baden, 1987, 113-136, hier 126.

27 Troberg, P.: Niederlassung, a. a. O., 936, Rz. 3.

28 Hailbronner, K./Nachbaur, A., a.a. O., 105.

29 Vgl. ebd., 108.

30 Hatzopoulos argumentiert, dass der EuGH - ähnlich wie auch bei der Warenverkehrsfreiheit - die Dienstleistungsfreiheit zunehmend auf Fälle ausdehnt, in denen das grenzüberschreitende Element kaum oder gar nicht ausgeprägt ist (Hatzopoulos, V., a.a.O., 58-62). Dies sei hier nur erwähnt, weil es sich noch lediglich um Tendenzen handelt.

31 Randelzhofer, A./Forsthoff, E.: Art. 49/50 EGV: Dienstleistungen, in: Grabitz, E./Hilf M. (Hg.): Das Recht der EU. Kommentar, München, 2001, Rz. 3 f., 40, 61.

32 EuGH, Urt. vom 30.11.1995, Rs. C-55/94 (Reinhard Gebhard/Consiglio dell'Ordine degli Avvocati e Procuratori di Milano), Slg. 1995 I, 4165, Rz. 39. 
freiheit relevant; das Tätigkeitsland wird zum Herkunftsland, und seine Regeln müssen angewandt werden. Würde die Dienstleistungsfreiheit nicht auf vorübergehende Tätigkeiten beschränkt, würde sie „auf einen Dienstleistungsaustausch zwischen Gebietsansässigen, mithin auf einen Inlandssachverhalt angewendet “. ${ }^{33}$

Diese Regulierungsbesonderheiten machen es sinnvoll, sich die bekannte Differenzierung zwischen Produkt- und Prozess-Standards zu vergegenwärtigen. ${ }^{34}$ Aufgrund der Nachfrage nach qualitativ hochwertigen Produkten wird durch die gegenseitige Anerkennung auf Produktstandards wenig Druck ausgeübt. Anders sieht es bei Gütern mit den Prozess-Standards aus. Das Ausmaß der durch seine Herstellung erzeugten Umweltverschmutzung sieht man Stahl nicht an, auch schlägt es sich im Produkt nicht nieder, ob in den Betrieben Mitbestimmung herrscht. ${ }^{35}$ Da sich produktionsbezogene Regeln direkt in den Kosten niederschlagen, ist es wahrscheinlicher, dass es in diesem Bereich zu einer Abwärtsspirale hin zu niedriger Regulierung kommt. Insofern bei Dienstleistungen Prozesse reguliert sind, folgt also im Vergleich zu Waren eine unterschiedliche Dynamik. Werden Prozesse harmonisiert oder einheitlich durch das Tätigkeitsland reguliert, wird Wettbewerbsdruck abgemildert. Gilt dagegen die gegenseitige Anerkennung und geht der Konsum mit der Erbringung von Dienstleistungen einher, treffen sehr unterschiedlich regulierte Prozesse aufeinander, und der Wettbewerbsdruck ist verschärft - so bei der Entsendung von Arbeitnehmern, wenn diese die Löhne ihres Herkunftslandes erhalten.

\section{Probleme des Binnenmarktes}

Die gegenseitige Anerkennung als Integrationsprinzip hat viele Vorteile. Es ist keine umständliche Vorab-Harmonisierung notwendig, es bleibt bei einer Regulierungsvielfalt und bei nationaler Zuständigkeit. Es handelt sich aber um ein Prinzip, das nicht absolut gilt und auf das sich die Mitgliedstaaten nur insoweit einlassen können, als ihnen bei berechtigten Interessen die Option verbleibt, den Handel eines Produktes auch abzuwehren. Die Abgrenzung zwischen berechtigten Interessen und verdecktem Protektionismus wird im Einzelfall immer umstritten bleiben. Insofern stößt schon der Binnenmarkt für Waren immer wieder

Scharpf, F.W.: Governing in Europe: Effective and Democratic?, Oxford, 1999, 91-101.

35 Ders.: Föderalismus und Demokratie in der transnationalen Ökonomie, in: Beyme, K. v./Offe, C. (Hg.):

Politische Theorien in der Ära der Transformation, Opladen, 1996, 211-235, hier 227. 
auf Schwierigkeiten und wird mit dem Vorwurf konfrontiert, dass er nicht richtig funktioniere. Auf bestehende Hindernisse des Binnenmarktes soll im Folgenden eingegangen werden, um danach die Besonderheit des Dienstleistungsbinnenmarktes zu erörtern. Aber nicht nur die gegenseitige Anerkennung führt zu Schwierigkeiten im Binnenmarkt. Auch die Mindestharmonisierung, die die gegenseitige Anerkennung komplettiert, wirft noch immer viele offene Fragen auf. Diese sollen zunächst kurz erörtert werden.

\section{Offene Fragen der Mindestharmonisierung}

Die Mindestharmonisierung soll in den Bereichen erfolgen, in denen die Mitgliedstaaten eigene Regeln nach dem Tätigkeitslandprinzip auferlegen können, gedeckt von den Ausnahmen von den Grundfreiheiten. Die Mindestharmonisierung ist aber Resultat eines politischen Verhandlungsprozesses. Scheitert dieser, erfolgt also Nicht-Einigung, gilt in diesem Bereich das Tätigkeitslandprinzip weiter, und der Markt bleibt fragmentiert. Um sich zu einigen, müssen manche Mitgliedstaaten also eventuell bereit sein, auf Höherregulierung zugunsten eines einheitlichen Marktes zu verzichten. Aber auch wenn die Mindestharmonisierung gelingt, bleibt offen, ob die vielfältigen Verhandlungen zu klaren Ergebnissen geführt haben. Erzielte Formelkompromisse sind interpretationsbedürftig, und der EuGH bezieht bei seiner Rechtsprechung neben dem unklar formulierten Sekundärrecht ebenso das Vertragsrecht ein.

Als besonders problematisch erweist sich die Frage der nationalen Höherregulierung. Sieht die Mindestharmonisierung keine abschließende Regelung des Schutzniveaus vor, können die Mitgliedstaaten höher regulieren. Es ist aber keineswegs einheitlich, für wen diese Höherregulierung gilt. Teils ist sie nur für inländische Produkte, teils aber auch für Importe zulässig, wenn die Richtlinie dazu explizit berechtigt, oder wenn sich dies implizit ergibt. ${ }^{36}$ Letzteres ist durchaus nicht unüblich. ${ }^{37}$ Innerhalb der Harmonisierung wiederholt sich also die Aufteilung in verschiedene Integrationsprinzipien: Neben der Totalharmonisierung gibt es bei der Mindestharmonisierung zum einen das Herkunftslandprinzip, wo nur für Inländer die Mindestvorgaben überschritten werden dürfen, und zum anderen das Tätigkeitslandprinzip, wo dies für In- und Ausländer geschehen kann. 
Ein Beispiel für eine Höherregulierung, die auch für ausländische Firmen gilt, ergibt sich aus Auseinandersetzungen um das deutsche Entsendegesetz. ${ }^{38}$ In verschiedenen Fällen ${ }^{39}$ ging es um die Verpflichtung der entsendenden Unternehmen, in den deutschen Urlaubsfonds einzuzahlen. Unter anderem war strittig, ob Deutschland ihnen eine längere Urlaubszeit vorschreiben konnte, als die bestehende Mindestharmonisierung nach einer Richtlinie von $1993^{40}$. Der EuGH urteilte, dass Deutschland seine höhere nationale Vorschrift nach dem Tätigkeitslandprinzip auch auf EU-ausländische Unternehmen ausdehnen dürfe. Damit urteilte der EuGH, dass eine bestehende Mindestharmonisierung nicht das Allgemeininteresse definiert; vielmehr können sich die Mitgliedstaaten auf die Ausnahme vom Vertragsrecht trotz existierender Mindestharmonisierung weiter berufen.

„There can be no mistake about the fact that this was a bit of a setback for the creation of the internal market. On the basis of this decision, every Member State can justify the restriction of fundamental freedoms by the public interest without causing a conflict with the object of the harmonization of laws. This effect contradicts the purpose and intent of the harmonization rules intended to make possible the creation of the internal market, i.e. the unrestricted exchange of persons, goods, services, and capital." ${ }^{41}$

Dougan hat anhand der Rechtsprechung des EuGH herausgearbeitet, dass die Frage, ob die Mindestharmonisierung für In- oder auch für Ausländer striktere nationale Regeln zulässt, vom EuGH äußerst widersprüchlich entschieden wird. "The overall impression generated by this body of case law thus remains one of confusion. “ ${ }^{42}$ In der Rechtsprechung sei keine klare Linie zu erkennen, so dass nur

Danwitz, T.v.: Die Rechtsprechung des EuGH zum Entsenderecht, in: Europäische Zeitschrift für Wirtschaftsrecht, $13 / 8$ (2002), 237-244.

39 Lohnausgleichskasse der Bauwirtschaft), Rs. C-50/98 (Urlaubs- und Lohnausgleichskasse der Bauwirtschaft/Amilcar Oliveira Rocha), Rs. C-52/98 (Urlaubs- und Lohnausgleichskasse der Bauwirtschaft/ Tudor Stone Ltd.), Rs. C-53/98 (Urlaubs- und Lohnausgleichskasse der Bauwirtschaft/Tecnamb-Tecnologia do Ambiante Ld ${ }^{\mathrm{a}}$ ), Rs. C-54/98 (Urlaubs- und Lohnausgleichskasse der Bauwirtschaft/Turiprata Construções Civil Lda), Rs. C-68/98 (Urlaubs- und Lohnausgleichskasse der Bauwirtschaft/Duarte dos Santos Sousa), Rs. 69/98 (Urlaubs- und Lohnausgleichskasse der Bauwirtschaft/Santos \& Kewitz Construções Lda), Rs. 7o/98 (Portugaia Construções Lda/Urlaubs- und Lohnausgleichskasse der Bauwirtschaft) und Rs. 71/98 (Engil Sociedade de Construção Civil SA/Urlaubs- und Lohnausgleichskasse der Bauwirtschaft), Slg. 2001 I, 7831.

40 RL 90/104/EG des Rates vom 23.11. 1993 über verschiedene Aspekte der Arbeitszeit (AblEU Nr. L 307/18 vom 13.12.1993).

41 Giesen, R.: Posting: Social Protection of Workers vs. Fundamental Freedoms?, in: Common Market Law Review, 40/1 (2003), 143-158, hier 156.

42 Dougan, M.: Minimum Harmonization and the Internal Market, in: Common Market Law Review, $37 / 4$ (2000), 853-885, hier 877; vgl. auch Herrnfeld, H.-H., a. a. O., 1195, Rz. 44. 
über einen Richterspruch des EuGH Sicherheit über die verbliebenen nationalen Kompetenzen zu erlangen sei. Auch die Rechtswissenschaft habe sich noch nicht zureichend mit dem Thema befasst. „Thus, although the general idea of minimum harmonization is enshrined in the Treaty and accepted by the Court, its full implications do not seem to have been thought out. “43

Ist die nationale Höherregulierung auch für ausländische Anbieter möglich, werden die Regulierungsinteressen gewahrt - das Ziel, mit der Mindestharmonisierung die Marktfragmentierung zu überwinden, wird aber verfehlt. Insofern scheint nur die Mindestharmonisierung unproblematisch, die eine Höherregulierung nur nach dem Herkunftslandprinzip erlaubt. Aber auch hier bestehen erhebliche Leerstellen.

Dies wird durch die Diskussion um die Inländerdiskriminierung verdeutlicht, die mit der nationalen, über europäische Mindeststandards hinausgehenden, Höherregulierung typischerweise einhergeht. Ein Beispiel ist die deutsche Versicherungsregulierung, bei der man auch nach der Implementierung der europäisch regulierten Finanzaufsicht die bis dahin ausgeübte Missstandsaufsicht des Bundesaufsichtsamtes für Versicherungswesen als nationale Höherregulierung beibehalten wollte. Daraufhin entspann sich eine juristische Diskussion darüber, inwieweit die damit verbundene Inländerdiskriminierung überhaupt zulässig sei. ${ }^{44}$ Unter anderem wurde argumentiert, man könne nicht mehr von der Verhältnismäßigkeit von Einschränkungen des Grundrechts der Berufsfreiheit (Art. 12 Abs. 1 GG) ausgehen, wenn ausländische Produkte ungehindert auf dem nationalen Markt Zugang hätten, die den Vorschriften nicht entsprächen.

Im Allgemeinen verletzt eine Inländerdiskriminierung das nationale Gleichheitsgebot, und Regulierungsauflagen erscheinen unverhältnismäßig. Höhere Auflagen sind schwer zu rechtfertigen, wenn politisch entschieden wurde, dass auch weniger regulierte Produkte keine Gefahr darstellen und zugelassen werden können. ${ }^{45}$ So kam es in Italien zu einer Aufhebung des Verbots, Nudeln mit Eigehalt herzustellen, nachdem einheimische Pastaproduzenten wegen Inländerdiskriminierung geklagt hatten und das Verfassungsgericht dies als Gleichheitsverstoß wertete. $^{46}$ Durch die Inländerdiskriminierung entsteht eine weitere nationale Deregulierungsdynamik.

43 Dougan, M., a.a. O., 885 .

44 Siehe z. B. Miersch, G.: Versicherungsaufsicht nach den Dritten Richtlinien, Karlsruhe, 1996, 169-72.

45 Weis, H.: Inländerdiskriminierung zwischen Gemeinschaftsrecht und nationalem Verfassungsrecht, in: Neue Juristische Wochenschrift, 36/48 (1983), 2721-2726, hier 2726.

46 Conant, L.: Justice Contained. Law and Politics in the European Union, Ithaca, N.Y./London, 2002, 14. 
„Even when States are authorised to impose higher requirements upon their nationals, Community minimum standards will in fact also tend to be the national maximum standard. “ 47

Damit ist die europäische Mindestharmonisierung mit der Option der nationalen Höherregulierung zwar erfolgreich als Möglichkeit, das Einigungsproblem auf europäischer Ebene zu erleichtern, bietet aber für berechtigte Regulierungsinteressen keine Lösung. Diese fallen leicht dem nationalen Gleichheitsgrundsatz zum Opfer. Diese Dynamik ist auch kaum abzumildern, wie das folgende Zitat zeigt:

„However, the protection of nationals, even to the disadvantage of nationals of other Member States, not only forms part of the concept of state sovereignty that the European Union has not completely destroyed, but is also a function of the State's existence. The existence of States, as identifiable political communities, only makes sense as long as those political communities express a greater degree of solidarity with their members than with non-members. This solidarity necessarily results in some sort of preference or discrimination in favour of members of that political community vis-à-vis the members of other political communities. “48

\section{Hindernisse des Binnenmarktes}

Seit Ende der 1990er Jahre hat die Kommission verschiedene Maßnahmen ergriffen, um das Funktionieren des Binnenmarktes zu verbessern, was auf die vielfältigen Hindernisse auch im Falle des Warenhandels hinweist. Hierbei geht es zum einen um die verbesserte Information über die Rechte und Pflichten der Markteilnehmer und mitgliedstaatlichen Behörden und zum anderen um das Bemühen, bei Konflikten unbürokratische Lösungen anzubieten, die zu einer Einigung führen, ohne dass es zunächst zu einem Vertragsverletzungsverfahren kommen muss. 1999 erließ die Kommission eine Mitteilung über die Anwendung der gegenseitigen Anerkennung, der zwei Zweijahresberichte über die auftretenden Probleme folgten. Eine weitere Mitteilung zur Auslegung des Prinzips erschien Ende 2003. Zudem wurden Sektorgespräche in Form Runder Tische zwischen den betroffenen Wirtschaftsakteuren und Behörden durchgeführt und einige Studien in Auftrag gegeben. ${ }^{49}$ Seit Juli 2002 ist das SOLVIT-Netzwerk in Betrieb, das bei Problemen mit dem Binnenmarkt eine unkomplizierte und rasche Problem-

47 Maduro, M. P., a. a. O., 135.

48 Ebd., 165, Hervorhebung im Original.

49 Für die einzelnen Dokumente siehe http://europa.eu.int/comm/internal_market/de/goods/mutrec.htm. 
lösung - „unterhalb“ des möglichen Wegs einer offiziellen Beschwerde bei der Europäischen Kommission oder des Beschreitens des Rechtswegs - verschaffen soll. Dafür wurden in den einzelnen Mitgliedstaaten Ansprechpartner bei den nationalen Verwaltungen eingerichtet, die innerhalb von zehn Wochen Abhilfe schaffen sollen. ${ }^{50}$

Die Beispiele, die bei SOLVIT angeführt werden, zeigen, dass Binnenmarktverpflichtungen vielfach verletzt werden. Aber es gibt auch immer wieder Fälle, die die Notwendigkeit von nationalen Ausnahmen verdeutlichen. So sind die sog. Magic Mushrooms in Großbritannien und den Niederlanden frei vermarktbar und können von dort in die Bundesrepublik Deutschland eingeführt werden. ${ }^{51}$ Heißt dies, dass ihre Vermarktung hier von den Binnenmarktfreiheiten gedeckt ist, oder kann die Bundesrepublik das Produkt eigenständig durch ein Verbot im Betäubungsmittelgesetz regulieren? Und wem obliegt die Beweislast im Falle eines Konflikts? Müsste die Bundesrepublik die Gefahren eines Konsums von Rauschpilzen rechtfertigen - schließlich gibt es Mitgliedstaaten, die zeigen, dass kein Verbot auch politisch zu rechtfertigen ist? Oder muss der Verkäufer darlegen, dass das Produkt so unbedenklich ist, dass die nationale Ausnahme von der Warenverkehrsfreiheit nicht greift?

\section{Schwierigkeiten des Dienstleistungsbinnenmarktes}

Wie für Waren gilt für Dienstleistungen, dass die Frage, wo genau die Grenze zwischen legitimen Regulierungsinteressen und Protektionismus verläuft, umstritten ist und sein muss. Hinzu treten einige Besonderheiten, die sich aus den Eigenschaften der Dienstleistungsregulierung, dem Dienstleistungshandel sowie der Dienstleistungsfreiheit des Vertrags erklären.

a) Die Mischung der Integrationsprinzipien und die vorübergehende Dauer der Dienstleistungsfreiheit

Selbst bei den Dienstleistungen, für die spezifische Richtlinien bestehen, konnte nicht vermieden werden, dass neben der Mindestharmonisierung und der gegenseitigen Anerkennung immer auch die Inländergleichbehandlung eine Rolle spielt. Damit wird die erzielte Vereinheitlichung des Marktes wieder geschmälert.

50 Vgl. http://europa.eu.int/solvit/.

51 Ich danke David Wildner für den Hinweis auf diesen Fall. 
So wird bei Finanzdienstleistungen die einheitliche Zulassung (sog. europäischer Pass) von den Mitgliedstaaten gegenseitig anerkannt. Der Souveränitätstransfer dieser Herkunftslandkontrolle lässt sich aber kompensieren, weil das Vertragsrecht des Tätigkeitslandes gilt; Unternehmen können also weit gehende regulative Erfordernisse weiterhin vorgeschrieben werden. Auch bei der Kabotage, der Dienstleistungsfreiheit im Straßengüterverkehr, wird die Zulassung gegenseitig anerkannt. Es gelten aber die Rechts- und Verwaltungsvorschriften des Aufnahmemitgliedstaates (so die Beförderungstarife und -bedingungen, Lenk- und Ruhezeiten und die Mehrwertsteuer). ${ }^{52}$

Die Steuerregelungen führen dazu, dass ein nationaler Steuerrepräsentant benannt werden muss. Zudem sind die Versicherungsvorschriften für Transporte in den Mitgliedstaaten uneinheitlich, was die ausreichende Versicherung der Waren erschwert. Diese Hindernisse führten in einer französischen Straßengüterverkehrszeitschrift zu der Frage: „En définitive, qui peut faire du cabotage?“" ${ }^{33}$ Außerdem ist die Kabotage auf Leistungen vorübergehender Dauer beschränkt, da sich die Dienstleistungsfreiheit nur auf temporäre Aktivitäten bezieht. Dauerhaft ist es unzulässig, beispielsweise von Luxemburg aus den deutschen oder französischen Markt zu bedienen. Diese europarechtlich bestehende Einschränkung der Dienstleistungsfreiheit wird aber sehr unterschiedlich wahrgenommen.

So heißt es beispielsweise in einer Bundestagsdrucksache, dass Transportunternehmen mit Gemeinschaftslizenz „unbegrenzt Kabotage betreiben“. Das GüKG schaffe „gleiches Recht für gebietsfremde Unternehmer aus anderen Mitgliedstaaten der EU/Vertragsstaaten des EWR (Kabotage) und gebietsansässige Unternehmen" 54 . Die Beschränkung auf die vorübergehende Natur der Dienstleistungserbringung wird also überhaupt nicht hervorgehoben. Dagegen betonen Verlautbarungen aus dem französischen Verkehrsministerium, dass regelmäßige Aktivitäten in Frankreich auch dort eine Niederlassung erfordern. Viele der 400 in Luxemburg niedergelassenen Unternehmen mit insgesamt 5.0oo Beschäftigten, zwei Drittel davon ursprünglich aus dem Ausland, würden diese europarechtlichen Bestimmungen nicht einhalten. ${ }^{55}$ Wirtschaftsrechts, München, 1998, 1-148, Nr. 176.

53 L'officiel des transporteurs magazine, 9/1999, 35.

54 Deutscher Bundestag: Effizienz des neuen güterkraftverkehrsrechtlichen Ordnungsrahmens, Unterrichtung durch die Bundesregierung, BT-Drs. 14/6906 (2001), 11, 5.

55 Délocalisations. 5 questions à Bernard Fournier, adjoint au DTT, in: La lettre du transport routier, 13.11. 2000-19. 11. 2000, 1. Meine Interviews bestätigen, dass in Deutschland anders als in Frankreich der eingeschränkte rechtliche Rahmen der Kabotage eigentlich nicht gesehen wird (Schmidt, S., a. a. O., Int. $18,21)$. 
b) Die gegenseitige Anerkennung der Kontrolle

Insofern die Erbringung und der Konsum von Dienstleistungen nicht getrennt sind, es sich also nicht um Korrespondenzdienstleistungen handelt, führt die gegenseitige Anerkennung der Kontrolle von Regulierung zu Schwierigkeiten. Bei Waren oder bei reinen Korrespondenzdienstleistungen kann der Mitgliedstaat, in dem die Produktion erfolgt und das Unternehmen niedergelassen ist, die Kontrolle der eigenen und der harmonisierten Regeln leisten. Anders ist es aber, wenn die Dienstleistungserbringung im Tätigkeitsland erfolgt. Hier kann die Zuständigkeit für die Regulierung und ihre Kontrolle auseinander fallen.

Besonders deutlich wird dies an der Finanzmarktregulierung, bei der die Rechtsaufsicht von der Finanzaufsicht zu trennen ist. Bei der Befolgung der gesetzlichen Vorschriften, der sonstigen Einhaltung des Geschäftsplans und der Wahrung der Belange der Versicherten handelt es sich weitgehend um Vorschriften des Tätigkeitslandes, die mit dem Allgemeininteresse gerechtfertigt werden. ${ }^{56}$ Nach Art. 28 der 3. Richtlinien ${ }^{57}$ wird den Mitgliedstaaten das Recht eingeräumt zu verhindern, dass Versicherungsverträge abgeschlossen werden, die im Widerspruch zu den geltenden Rechtsvorschriften des Allgemeininteresses stehen. Hier$\mathrm{zu}$ hat die deutsche Aufsichtsbehörde eine längere Liste veröffentlicht, in der allgemein auf verschiedene Gesetze, etwa gegen den unlauteren Wettbewerb, das BGB und unterschiedliche Pflichtversicherungsgesetze verwiesen wird. Die Aufsicht hierüber obliegt weitgehend der Herkunftslandaufsicht, allerdings ist die Tätigkeitslandbehörde subsidiär zuständig.

In Deutschland darf das Aufsichtsamt bei inländischen Unternehmen unmittelbar im Rahmen der Missstandsaufsicht einschreiten. Ausländische Unternehmen kann die Tätigkeitslandbehörde nach Art. 40 Abs. 3 der 3. Richtlinien auffordern, Unregelmäßigkeiten abzustellen. Geschieht dies nicht, muss die Herkunftslandbehörde gebeten werden, die erforderlichen Maßnahmen zu treffen. Bleibt diese untätig, kann die Tätigkeitslandbehörde in begrenztem Rahmen einschreiten. ${ }^{58}$ Kommt es zum Rechtsstreit, sind die Gerichte des Herkunftslandes

56 Criegern, U.v.: Die Bedeutung der Dritten Schadenrichtlinie unter aufsichtsrechtlichen Gesichtspunkten für die Dienstleistungsfreiheit der Versicherungsunternehmen in dem Binnenmarkt, Frankfurt/M. u. a., 1997, 157, 160, 171; Miersch, G., a. a. O., 73-75.

57 RL 92/96/EWG des Rates vom 10.11. 1992 zur Koordinierung der Rechts- und Verwaltungsvorschriften für die Direktversicherung (Lebensversicherung) sowie zur Änderung der Richtlinien 79/267/EWG und 90/619/EWG (Dritte Richtlinie Lebensversicherung, AblEU Nr. L 360/1 vom 09. 12.1992); RL 92/49/EWG des Rates vom 18.06.1992 zur Koordinierung der Rechts- und Verwaltungsvorschriften für die Direktversicherung (mit Ausnahme der Lebensversicherung) sowie zur Änderung der Richtlinien 73/239/ EWG und 88/357/EWG (Dritte Richtlinie Schadenversicherung, AblEU Nr. L 228/1 vom 11. 08.1992).

58 Andreae, P.: Industrieversicherung und einzelne Versicherungsarten, in: Schwappach, J. (Hg.): EURechtshandbuch für die Wirtschaft, München, 1996, 126-144, hier 129. 
zuständig. Sie müssen ihrer Entscheidung dann das Recht des Tätigkeitslandes zugrunde legen. ${ }^{59}$ Diese Aufteilung der Zuständigkeiten führte bei der deutschen Aufsicht früh zu Befürchtungen:

„Ich halte mich aber als Leiter der deutschen Aufsichtsbehörde nicht für kompetent genug, um festzustellen, ob deutsche Versicherungsunternehmen bei ihrer Versicherungstätigkeit beispielsweise in Griechenland oder Belgien oder in einem anderen europäischen Staat die dortigen zwingenden nationalen Vorschriften einhalten. “60 „Somit wird letztlich das BVerwG zu entscheiden haben, ob das VU [Versicherungsunternehmen, S.K.S.] zwingende ausländische Rechtsvorschriften, und zwar regelmäßig zivilrechtliche Vorschriften, verletzt. Sehr befriedigend ist dieses Ergebnis nicht." 61

Da Prüfungen für die Finanzaufsicht allein dem Herkunftsmitgliedstaat obliegen, ergibt sich auch die Zuständigkeit für Zweigniederlassungen in anderen Mitgliedstaaten. Damit werden hoheitliche Befugnisse in anderen Mitgliedstaaten ausgeübt. ${ }^{62}$

Durch die Herkunftslandkontrolle sind die Aufsichtsbehörden auf enge Zusammenarbeit angewiesen. Hierzu gibt es in den Richtlinien verschiedene Verpflichtungen. Bisher ist die Erfahrung weitgehend positiv, allerdings kam es aufgrund der beschränkten Nutzung der Dienstleistungsfreiheit noch zu wenigen Konflikten, so dass auch zu den Regelungen des Gerichtsstands mit den oben angemerkten Befürchtungen die Erfahrungen fehlen. ${ }^{63}$ Im Dienstleistungsbinnenmarkt verschärfen sich somit die Probleme der Herkunftslandkontrolle.

„Home State control may in principle constitute a more efficient market-building device than host State control, but there are problems associated with the plausibility of home State respect for the foundational rules of the European re-regulatory bargain that are directly connected to the ambitiously dexterous constitutional sleight of hand that treats national agencies, anchored to a political chain of State-focused responsibility and accountability, as responsible for the enforcement of Community rules governing the European market." 64 Die Versicherungsrundschau, 46/11 (1991), 329-341, hier 332.

61 Hohlfeld, K.: Veränderte Aufgaben für das Bundesaufsichtsamt infolge der Deregulierung, in: Kalwait, R./Bald, E.-J. (Hg.): Versicherungswirtschaft im Umbruch, Karlsruhe, 1995, 1-15, hier 5.

62 Fahr, U.: Die Umsetzung der Versicherungsrichtlinien der dritten Generation in deutsches Recht, in: Versicherungsrecht, 43/25 (1992), 1033-1047, hier 1035.

63 Interviewinformation (Schmidt, S., a. a. O., Int. 7, 19, 22).

64 Weatherill, S.: Pre-emption, Harmonisation and the Distribution of Competence to Regulate the Internal Market, in: Barnard, C./Scott, J. (Hg.): The Law of the Single European Market, Oxford, 2002, 41-73, hier 68, Hervorhebung im Original. 
Die Dienstleistungsfreiheit zielt auf grenzüberschreitende Aktivitäten. Reine Inlandssachverhalte werden in der Regel nicht erfasst. Dennoch hat die Realisierung der Dienstleistungsfreiheit zur Folge, dass weniger der Wettbewerb im Binnenmarkt, sondern der Wettbewerb auf nationalen Märkten zunimmt. Inwieweit nationale Liberalisierungswirkungen erfolgen, ist dabei abhängig vom Ausmaß der bestehenden nationalen Regulierung.

Hinter den möglichen erheblichen nationalen Liberalisierungswirkungen steht der spezifische Regulierungsansatz im Binnenmarkt, der sich nicht auf die Ermöglichung grenzüberschreitenden Handels beschränkt. Stattdessen wird ein umfassender Koordinierungsansatz verfolgt, der sich sowohl an rein national als auch an grenzüberschreitend tätige Firmen richtet. Wie das folgende Zitat zeigt, verhindert man auf diese Weise sowohl Wettbewerbsverzerrungen zwischen unterschiedlich tätigen Unternehmen als auch die Erschwernis, wenn grenzüberschreitender Handel nur unter zusätzlichen Auflagen möglich wird.

„Es wäre daher theoretisch denkbar, eine Koordinierungsrichtlinie nur für solche Unternehmen zu erlassen, die bereits grenzüberschreitend tätig sind oder dies noch werden wollen. Problematisch ist an diesem Ansatz bereits, dass potentiell jedes in der EG ansässige Unternehmen grenzüberschreitend tätig werden kann, was zu Abgrenzungsschwierigkeiten führt. Durch die Parallelität von zwei Rechtssystemen würde außerdem die Wettbewerbssituation zwischen Unternehmen, die ihren Sitz in demselben Mitgliedstaat haben, verzerrt. Schließlich führte die Differenzierung zu unerwünschten Hindernissen im Falle des Gebrauchs der Freiheiten. Entschiede sich ein Unternehmen erst später zu einer grenzüberschreitenden Tätigkeit, so ginge damit ein Wechsel der anwendbaren Rechtsnormen einher; es wäre denkbar, dass ein Unternehmen zunächst bestimmte, durch die ,europäischen Rechtsnormen' aufgestellte Anforderungen erfüllen müsste, was mit dem Gedanken des Binnenmarktes kaum zu vereinbaren ist." 65

So hatte die Realisierung der Dienstleistungsfreiheit in Deutschland einen deutlichen Liberalisierungseffekt für die Versicherungsregulierung; Gleiches gilt für die europäische Regulierung des Straßengüterverkehrs. Diese weit reichenden nationalen Auswirkungen kontrastieren mit der geringen Nutzung des neuen Regulierungsrahmens für grenzüberschreitenden Dienstleistungshandel. So betrug der Anteil der Kabotage am gesamten EU-Straßengüterverkehr 2001 nur $0,76 \%$. Die meisten Kabotagedienstleistungen wurden in Deutschland und Frankreich erbracht; dort hatten sie einen Marktanteil von 1,6\% bzw. 1,1\%. ${ }^{66} \mathrm{Im}$ Versicherungsmarkt hatten im Jahr 2000 Unternehmen, die unter der Herkunfts-

65 Miersch, G., a. a. O., $137 \mathrm{f}$.

66 Vgl. Oberhausen, J.: Le cabotage routier de 1999 à 2001, Eurostat Statistiques en bref - Transports 7/2003. 
landaufsicht im EU-Ausland standen, einen Marktanteil von 3,2 \% in Deutschland. Dagegen hatten ausländisch kontrollierte Unternehmen (mindestens 50 Prozent), die als Niederlassungen in Deutschland reguliert sind, einen Marktanteil von $13 \%{ }^{67}$ Ausländische Niederlassungen sind also deutlich wichtiger - hierfür bräuchte man aber nicht eine so weitgehende Vereinheitlichung der Aufsichtsprinzipien.

Mit der Harmonisierung kommt es zu einem Souveränitätstransfer auf die europäische Ebene. Auch diese Problematik wird bisher in der Literatur zur europäischen Integration kaum diskutiert. Die große Bedeutung europäischen (Sekundär-)Rechts im nationalen Kontext lässt sich auch an der Tätigkeit des EuGH ablesen, der vielfach über rein nationale Wirtschaftstätigkeiten urteilen muss, da europäisches Recht aufgrund des einheitlichen Ansatzes maßgeblich ist. So muss der EuGH beispielsweise über die Frage der Einhaltung der Arbeitszeiten von Omnibus- oder Lastkraftwagenfahrern entscheiden, auch wenn diese ausschließlich im innerstaatlichen Verkehr tätig waren, da diese Arbeitszeiten durch eine europäische Verordnung reguliert sind. ${ }^{68}$

Auch ein Abrücken von dem umfassenden Ansatz der Harmonisierung wäre keine Option, selbst wenn man grundsätzlich an einer doppelten Rechtsordnung für rein national und grenzüberschreitend agierende Unternehmen nichts auszusetzen hätte. Dies ist offensichtlich, wenn man bedenkt, auf welchem Regulierungsniveau eine solche reine Binnenmarktordnung anzusiedeln wäre. Handelte es sich um eine hohe Setzung, wären solcherart regulierte Unternehmen in Ländern mit niedrigen Standards gegenüber einheimischen Unternehmen benachteiligt, deren Märkte also quasi abgeschottet. Bei niedriger Setzung käme es dagegen zu einer Inländerdiskriminierung gegenüber Unternehmen in hoch regulierenden Mitgliedstaaten, die dann in ihrem Heimatmarkt gegenüber EUAusländern nicht konkurrenzfähig sein könnten.

67 Bundesanstalt für Finanzdienstleistungsaufsicht: Geschäftsbericht 2001, Teil B, Bonn, 2003, 6; OECD: OECD Statistics, Financial and Fiscal Affairs, Insurance Statistics, Paris, 2003, C3131-C3112.

68 EuGH, Urt. vom 02.10.1991, Rs. C-7/90 (Strafverfahren Vandevenne u. a.), Slg. 1991 I, 4371; Urt. vom 02. 10.1991, Rs. C-8/90 (Strafverfahren Kennes u. a.), Slg. 1991 I, 4391; Urt. vom 25. 06.1992, Rs. C-116/91 (Licensing Authority South Eastern Traffic Area/British Gas plc.), Slg. 1992 I, 4071; Urt. vom 15.12. 1993, Rs. C-116/92 (Strafverfahren Charlton u.a.), Slg. 1993 I, 6755; Urt. vom 09. 06.1994, Rs. C-394/92 (Strafverfahren Michielsen u. a.), Slg. 1994 I, 2497; Urt. vom 09.11.1995, Rs. C-235/94 (Strafverfahren Bird), Slg. 1995 I, 3933; Urt. vom 21. 03.1996, Rs. C-335/94 (Mrozek u.a.); Slg. 1996 I, 1573; Urt. vom 28. 09. 2000, Rs. C-103/99 (Strafverfahren Hume), Slg. 2000 I, 7809. 


\section{Fazit}

Die gegenseitige Anerkennung und die Mindestharmonisierung, auf denen der Binnenmarkt als Kernstück der Union beruht, beinhalten noch überraschend viele Unwägbarkeiten und offene Fragen. Diese sollen abschließend zusammengefasst und auch im Hinblick auf den momentan breit diskutierten Vorschlag für eine Dienstleistungsrichtlinie, mit dem die Kommission die Dienstleistungs- und Niederlassungsfreiheit für alle noch nicht speziell regulierten Dienstleistungen auf einen Schlag verwirklichen will, erörtert werden. Dabei soll die zentrale These des Beitrags, dass die mit der gegenseitigen Anerkennung verwirklichte Integration notwendigerweise unvollkommen ist, es hierzu aber dennoch keine Alternativen gibt, näher ausgeführt werden.

Bei der Mindestharmonisierung ist ungeklärt, wie das Recht der Mitgliedstaaten auf Höherregulierung gegen Wettbewerbsdruck gesichert werden kann - ohne dass daraus eine erneute Marktfragmentierung resultiert. Bei der gegenseitigen Anerkennung der Kontrolle erscheint ungewiss, inwieweit die Umwidmung nationaler Behörden in europäische gelingt. Dabei zeigt die Herkunftslandkontrolle von Tätigkeitslandregeln, die bei Dienstleistungen weiter eine große Rolle spielen, wie viele Probleme es gäbe, wäre der Binnenmarkt für grenzüberschreitende Dienstleistungen nicht ein nur marginales Phänomen. Schließlich macht der einheitliche Ansatz bei der Harmonisierung deutlich, wie sehr es zu einer Privilegierung des Binnenmarkts gegenüber inländischen Sachverhalten kommt. Selbst wo nur eine geringe Nutzung erwartbar ist, werden für diese umfassende nationalstaatliche Reformen und eine weit reichende Kompetenzübertragung in Kauf genommen.

Deutlich wird am Binnenmarkt für Dienstleistungen, wie oft auf Regulierungen des Tätigkeitslandes zurückgegriffen werden muss. Da Dienstleistungsregulierung oft Prozessregulierung ist, die sich nur schwer harmonisieren lässt, bleibt keine andere Wahl. Mit der Inländergleichbehandlung wird gleichzeitig der Souveränitätstransfer durch die gegenseitige Anerkennung und die Harmonisierung kompensiert. Selbst der momentan umstrittene Vorschlag für eine Dienstleistungsrichtlinie greift für Verträge mit Endverbrauchern auf das nationale Vertragsrecht zurück. Inwieweit sich hierdurch die Deregulierungseffekte der Richtlinie kompensieren lassen, wird bisher in der Diskussion noch nicht zureichend beachtet.

Mit der gegenseitigen Anerkennung bzw. dem Herkunftslandprinzip kann der Binnenmarkt nur unvollkommen verwirklicht werden. Dafür ist es wichtig, sich zu vergegenwärtigen, dass die Prinzipien des Cassis-de-Dijon Urteils vom EuGH ${ }^{69}$ 
nicht nur parallel zu einer Erweiterung der Ausnahmen von der Warenverkehrsfreiheit eingeführt wurden, sondern auch zu einer Zeit, als es lediglich neun relativ homogene Mitgliedstaaten gab. Diese Ausgangsbedingung hat sich mit den Süderweiterungen und der Osterweiterung signifikant verändert. Die gewachsene Heterogenität der Mitgliedstaaten steht einer parallelen Ausweitung des Herkunftslandprinzips entgegen und legt eher eine Einschränkung aufgrund des verringerten Vertrauens zwischen den Mitgliedstaaten nahe. Der mit der gegenseitigen Anerkennung einhergehende horizontale Souveränitätstransfer kann in einer immer heterogeneren Union nur schlechter funktionieren, weil man nicht auf einen ausreichenden Schutz der eigenen Bevölkerung vertrauen kann, wenn die Bedingungen in den Herkunftsländern unbekannt sind, und diese tatsächlich auch stärker differieren.

Dennoch stellt auch die Harmonisierung keinen Ausweg dar. Mit der Harmonisierung geht ein Souveränitätstransfer einher, der mit dem Problem behaftet ist, dass die einmal vereinbarte Regulierung aufgrund der anspruchsvollen Entscheidungsregeln in der Union (vergleichbar verfassungsändernden Mehrheiten auf der nationalen Ebene) nur äußerst schwer geändert und angepasst werden kann. Zudem führt die umfassende Vereinheitlichung der Rechtsordnungen dazu, dass etwa der EuGH letztinstanzlich entscheiden muss, ob ein Omnibusfahrer bei einer rein nationalen Fahrt die Arbeitszeiten korrekt eingehalten hat, weil es sich um eine europäische Verordnung handelt.

Die gegenseitige Anerkennung - das zeigen die Alternativen der Harmonisierung und des Tätigkeitslandprinzips deutlich - ist deshalb ein unverzichtbarer Ansatz für den Binnenmarkt. Die Unsicherheiten über das Ausmaß der Marktfreiheiten und der verbleibenden nationalen Kompetenzen sind dabei unumgängliche Reibungsverluste und gegenüber den Nachteilen der bestehenden Alternativen ein kleineres Übel. Gerade für den Dienstleistungsbinnenmarkt gilt es zudem zu beachten, dass dieser aufgrund der Formulierung der Dienstleistungsfreiheit als Residualkategorie eine marginale Aktivität darstellt. Beschränkt auf eine vorübergehende Dauer - die gerade diskutierte Berufsqualifikationsrichtlinie definiert dies als 16 Wochen $^{70}$ - ist die (legale) grenzüberschreitende Dienstleistungserbringung wohl eher die Ausnahme als die Regel. Die gerade bei Dienstleistungen wichtige Einstellung auf Kundenwünsche, das notwendige Vertrauen, die Bedeutung von Reputation und die Sprachbarrieren - zusammen mit Kenntnissen über noch bestehende Vorschriften des Tätigkeitslandes - machen eine vorübergehende Dienstleistungserbringung nur in Ausnahmen attraktiv. Niederlassungen sind deshalb wichtiger, und auch für dauerhaft grenzüberschreitend 
erbrachte Dienstleistungen ist der Regulierungsrahmen des Tätigkeitslandes der notwendige Maßstab. ${ }^{71}$

Auch der vorliegende Vorschlag der Dienstleistungsrichtlinie, mit der sich die Mitgliedstaaten sekundärrechtlich dem Herkunftslandprinzip verpflichten sollen, bei einer gleichzeitigen näheren Umschreibung und Begrenzung der Ausnahmen im Allgemeininteresse, bei denen das Tätigkeitslandprinzip gilt, geht über die Implikationen der Beschränkung auf vorübergehende Tätigkeiten hinweg. Die bisher geringen Marktanteile des grenzüberschreitenden Dienstleistungsverkehrs lassen sich aber gut mit dieser vertragsrechtlichen Beschränkung erklären. Die Kommission setzt dagegen geringen grenzüberschreitenden Dienstleistungshandel mit unzulässigen nationalen Beschränkungen gleich und leitet hieraus, auch beim Finanzdienstleistungsbinnenmarkt ${ }^{72}$, die Notwendigkeit zusätzlicher politischer Maßnahmen ab. Ob für vorübergehende Tätigkeiten ein weiterer Souveränitätstransfer mit einer Umstellung auch der nationalen Sektorregulierung sinnvoll ist, bleibt dabei weitgehend unbeachtet. Ohne eine Diskussion über die Möglichkeiten und Grenzen der Förderung des Dienstleistungsbinnenmarktes angesichts des Fokus auf vorübergehende Tätigkeiten bleibt zu befürchten, dass der vorherrschende Effekt einer Dienstleistungsrichtlinie tatsächlich in der momentan breit diskutierten nationalen Deregulierung und weniger in vermehrtem "echten“ Dienstleistungshandel bestünde.

Über die Dienstleistungsrichtlinie muss noch im Ministerrat und im Europäischen Parlament entschieden werden. Angesichts der Interessenunterschiede zwischen den Mitgliedstaaten mit hohen Lohnkosten, die sich als Empfänger von Dienstleistungen sehen, und jenen mit niedrigen Lohnkosten, die die Dienstleistungsfreiheit nutzen wollen, ist nur die Einigung auf eine modifizierte Richtlinie denkbar. Die Rückfallposition bei Nicht-Einigung stellt hierbei die Rechtsprechung des EuGH dar, über die die Dienstleistungsfreiheit alternativ verwirklicht werden kann. Da der EuGH den Mitgliedstaaten bisher bei der Dienstleistungsfreiheit einen relativ breiten Handlungsspielraum zugemessen hat, sollten die Mitgliedstaaten, die Vorbehalte gegen die Richtlinie vorbringen, eine recht gute Verhandlungsposition haben.

Aufgrund der Besonderheiten des Dienstleistungshandels - des häufigen $\mathrm{Zu}$ sammenfallens von Erbringung und Konsum - wirft eine breite Anwendung des Herkunftslandprinzips besondere Kontrollprobleme auf und lädt zur Umgehung

71 Roth, W.-H.: The European Court of Justice's Case Law, a.a. O., 20.

72 Gkoutzinis, A.: Free Movement of Services in the EC Treaty and the Law of Contractual Obligations Relating to Banking and Financial Services, in: Common Market Law Review, 41/1 (2004), 119-175, hier 121. 
nationaler Regulierung ein. Wie aufgezeigt, bedarf die gegenseitige Anerkennung für ihr Funktionieren aber eines breiten Vertrauens und einer solidarischen Orientierung der Beteiligten. Führt der forcierte Dienstleistungshandel vor allem zur Umgehung nationaler Regulierung - und damit zu einer „egoistischen“ Orientierung an den individuellen Vorteilen eines Regulierungswettbewerbs -, ist es äußerst zweifelhaft, ob der Binnenmarkt unter diesem Vorzeichen dauerhaft funktionieren kann. 